Rochester Institute of Technology

RIT Scholar Works

Presentations and other scholarship

Faculty \& Staff Scholarship

$11-1-2019$

\title{
Addressing the Accessibility of Social Media
}

Cole Gleason

Carnegie Mellon University

Patrick Carrington

Carnegie Mellon University

Lydia B. Chilton

Columbia University

Benjamin M. Gorman

Bournemouth University

Hernisa Kacorri

University of Maryland

See next page for additional authors

Follow this and additional works at: https://scholarworks.rit.edu/other

\section{Recommended Citation}

Cole Gleason, Patrick Carrington, Lydia B. Chilton, Benjamin M. Gorman, Hernisa Kacorri, Andrés MonroyHernández, Meredith Ringel Morris, Garreth W. Tigwell, and Shaomei Wu. 2019. Addressing the Accessibility of Social Media. In Conference Companion Publication of the 2019 on Computer Supported Cooperative Work and Social Computing (CSCW '19). Association for Computing Machinery, New York, NY, USA, 474-479. DOI:https://doi.org/10.1145/3311957.3359439

This Conference Proceeding is brought to you for free and open access by the Faculty \& Staff Scholarship at RIT Scholar Works. It has been accepted for inclusion in Presentations and other scholarship by an authorized administrator of RIT Scholar Works. For more information, please contact ritscholarworks@rit.edu. 


\section{Authors}

Cole Gleason, Patrick Carrington, Lydia B. Chilton, Benjamin M. Gorman, Hernisa Kacorri, Andres MonroyHernandez, Meredith Ringel Morris, Garreth W. Tigwell, and Shaomei Wu 


\section{Addressing the Accessibility of Social Media}

\section{Cole Gleason}

Patrick Carrington

Carnegie Mellon University

\{cgleason,pcarrington\}@cmu.edu

\section{Benjamin M. Gorman}

Bournemouth University

bgorman@bournemouth.ac.uk

Andrés Monroy-Hernández

Snap Inc.

amh@snap.com

\section{Garreth W. Tigwell}

Rochester Institute of Technology

garreth.w.tigwell@rit.edu

\author{
Lydia B. Chilton \\ Columbia University \\ chilton@cs.columbia.edu
}

\author{
Hernisa Kacorri \\ University of Maryland \\ hernisa@umd.edu
}

\section{Meredith Ringel Morris \\ Microsoft Research}

merrie@microsoft.com

\author{
Shaomei Wu \\ Facebook \\ shaomei@fb.com
}

\section{ABSTRACT}

Social media platforms are deeply ingrained in society, and they offer many different spaces for people to engage with others. Unfortunately, accessibility barriers prevent people with disabilities from fully participating in these spaces. Social media users commonly post inaccessible media, including videos without captions (which are important for people who are deaf or hard of hearing) and images without alternative text (descriptions read aloud by screen readers for people who are blind). Users with motor

Permission to make digital or hard copies of part or all of this work for personal or classroom use is granted without fee provided that copies are not made or distributed for profit or commercial advantage and that copies bear this notice and the full citation on the first page. Copyrights for third-party components of this work must be honored. For all other uses, contact the owner/author(s).

CSCW '19 Companion, November 9-13, 2019, Austin, TX, USA

() 2019 Copyright held by the owner/author(s).

ACM ISBN 978-1-4503-6692-2/19/11.

https://doi.org/10.1145/3311957.3359439 

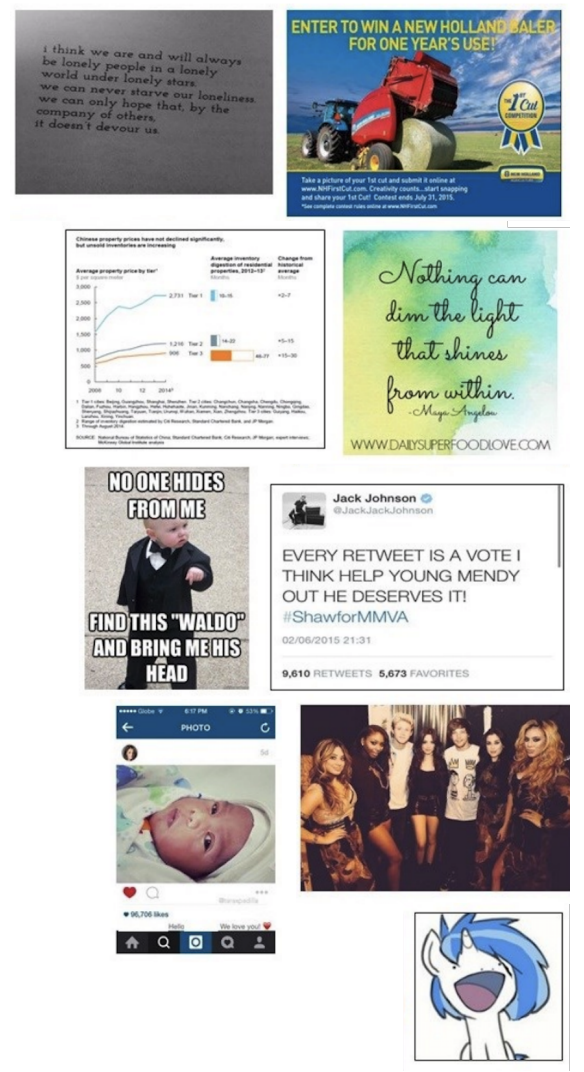

Figure 1: Common types of image content on Twitter that lack descriptions (as detailed in [16]). Left column: picture of text, graph, meme, screenshot. Right column: image with embedded text (ad), quotation, unofficial retweet, photograph, drawing. impairments must find workarounds to deal with the complex user interfaces of these platforms, and users with cognitive disabilities may face barriers to composing and sharing information.

Accessibility researchers, industry practitioners, and end-users with disabilities will come together to outline challenges and solutions for improving social media accessibility. The workshop starts with a panel of end-users with disabilities who will recount their Perspectives of Successes and Barriers. Industry professionals from social media companies (e.g., Facebook and LinkedIn) will detail their Design Process and Implementation Challenges in a panel with questions from attendees. The attendees will share their work and tackle Open Challenges and Future Research Directions. This workshop will forge collaborations between researchers and practitioners, and define high-priority accessibility challenges for social media platforms.

\section{ACM Reference Format:}

Cole Gleason, Patrick Carrington, Lydia B. Chilton, Benjamin M. Gorman, Hernisa Kacorri, Andrés MonroyHernández, Meredith Ringel Morris, Garreth W. Tigwell, and Shaomei Wu. 2019. Addressing the Accessibility of Social Media. In 2019 Computer Supported Cooperative Work and Social Computing Companion Publication (CSCW'19 Companion), November 9-13, 2019, Austin, TX, USA. ACM, New York, NY, USA, 6 pages. https://doi.org/ $10.1145 / 3311957.3359439$

\section{WORKSHOP DESCRIPTION}

Social media platforms have quickly evolved from niche online gathering spaces to teeming public forums for all manner of discourse. These spaces host critical discussions regarding international news, local happenings, and activism, yet they are often inaccessible to people with disabilities. As a society, we have used both policy and technology to make physical and online spaces accessible, but social media platforms present unique challenges for accessibility that remain unsolved. The high prevalence of user-generated content [4] makes it harder to ensure images and videos are accessible to users with vision or hearing impairments. The interfaces for social media platforms are more complex than many standard websites, and people with vision, motor, or cognitive impairments find them difficult to use.

Recent research observing social media platforms and speaking to users with disabilities about their interactions with social media has revealed that these platforms often become more inaccessible over time. For example, as of June 2015, the increase of graphical media present on Twitter resulted in $28.4 \%$ of content being inaccessible to blind users [16]. Twitter has tried to address this problem by asking users to add alternative text descriptions to their tweet images, but only $0.1 \%$ of images currently have this [7]. Even content surrounding accessibility topics, such as the \#HandsOffMyADA campaign, only have $6.7 \%$ described images and $2.4 \%$ captioned videos [1]. In contrast, other high-traffic websites often have alternative text on $40 \%$ to $70 \%$ of images $[2,8]$. Social media platforms have the clout and potential impact to substantially improve content accessibility by introducing new standards and 


\section{ORGANIZER BACKGROUNDS}

Cole Gleason is a Ph.D. student at Carnegie Mellon University who develops assistive technology for people with vision impairments. His research focuses on navigation tools for physical spaces, as well as accessibility tools available on social media platforms. Website: http://colegleason.com

Patrick Carrington is an Assistant Professor at Carnegie Mellon University. His research focuses on augmenting interactive assistive technologies, like wheelchairs, to support the broad needs of people with disabilities. Recently he has been exploring the accessibility of media and imagery on the web. Website: http://patrickcarrington.com

Lydia B. Chilton is an Assistant Professor at Columbia University. Her research is on computational design and how meaning is conveyed through images and humor, which are often shared online. She is broadening her research to include how this meaning can also be shared in accessible ways. Website: http: //www.cs.columbia.edu/ chilton

Benjamin Gorman is a Lecturer in Computer Science at Bournemouth University, and his research focuses on technology to help people with hearing loss. Recently he has been studying accessibility challenges faced by people with visual impairments interacting with emoji on social media, and personalizing the user experience of subtitles on streaming websites. Website: http://benjgorman.com

Hernisa Kacorri is an Assistant Professor at University of Maryland, College Park. She leads the Intelligent Assistive Machines Lab, a research group working at the intersection of artificial intelligence and accessibility. Her research has previously studied how the disability community mobilizes through social media for advocacy, activism, and social change. Website: http://hernisakacorri.com approaches. Morris and colleagues explored the design of alternative text for images, finding that it has been stagnant since its introduction in 1995, and that richer representations can be designed to take advantage of modern computational abilities [15]. Facebook and Instagram have recently introduced automatic description of images [21], leading to high coverage, although users must have high trust in these automatic systems to add value [14].

Although platforms struggle to ensure their user-generated content is accessible, their interface design is more under their control and can have a comparable impact. Researchers have found that although blind Facebook users are able to participate in many of the platform's core social activities, including posting status updates and sharing photos [19], they also hit usability barriers completing other core tasks, including creating a user profile or searching for other users [3]. Researchers also found that when blind people reach accessibility barriers, they either find non-standard workarounds (i.e., using the mobile interface on a desktop computer), or avoid the inaccessible features entirely.

Prior research with people with physical or communication disabilities who access Twitter through augmentative and alternative communication (AAC) devices found that these users enjoy social media platforms in close-knit networks and exhibit different posting and sharing behavior that might be linked to their method of accessing the platform [9-11].

Interface design presents an opportunity for social media platforms to assist people with disabilities, such as ensuring graphics and icons used are readable and easy to understand [17]. For example, researchers found that Facebook users with dyslexia had difficulties with reading content on the site, but especially with writing content [18]. The researchers then designed and evaluated a writing assistance tool to help proofread their writing when posting [20]. The tool increased the confidence of the participants with dyslexia, showing how integration of this types of tools can improve the experience for many users with dyslexia.

While everyone should be able to access community, news, and other content on social media platforms, people with disabilities often rely on these networks for critical activism and support. On Twitter, groups of disability advocates have convened around hashtags like \#CripTheVote [5] and \#HandsOffMyADA [1] to organize political support of disability legislation. Online activism also can be a more accessible outlet for political advocacy than physical marches or protests, so people with a disability may turn to virtual activism more often [12]. These platforms are also a place to seek social support online about accessibility barriers and solutions [13]. Campaigns like \#SayTheWord use social media to change the public perception and awareness of people with disabilities, calling for a unified identity and a recognition of a critical constituency of people (25\% of US adults [6]).

Because social media platforms are an important venue for both daily communication and disability activism, barriers must be reduced so that they are accessible to all. This workshop brings together key stakeholders to discuss interface and content barriers, create design guidelines to dissolve these barriers, and propose research agendas to address the challenges in this space. We aim to have 


\section{ORGANIZER BACKGROUNDS (CONT.)}

Andrés Monroy-Hernández is a Lead Research Scientist at Snap Inc. where he manages the Human Computer Interaction team focus ing on building new technologies that enable people to connect and collaborate. He is new to the accessibility field but is excited by the idea of broadening access to social technologies. Website: http://andresmh.com

Meredith Ringel Morris is a Principal Researcher at Microsoft Research, where she manages the Ability research group, focusing on accessible technologies. She has studied accessibility challenges faced by blind users of Twitter, and challenges experienced by sign language speakers using social media. Website: http://aka.ms/merrie

Garreth Tigwell is an Assistant Professor at the Rochester Institute of Technology. Garreth researches situational impairments and supporting designers with tools to improve app $\&$ web accessibility. Garreth previously investigated the communication challenges presented by emoji, which are commonly used on social media, and he is now investigating their inaccessibility. Website: http://garrethtigwell.com Shaomei $\mathbf{W u}$ is a researcher scientist in Facebook $\mathrm{Al}$, where she leads the $\mathrm{Al}$ for inclusion initiative to build Al-based technologies for people from marginalized and under-resourced communities. She previously worked at the Computational Social Science team at Facebook where she built temporal and structural models to study how information and behavior spread in the network. researchers, practitioners, and the general public to benefit from the outcomes of this workshop, hoping to reach everyone who can make large strides in addressing these accessibility challenges.

\section{WORKSHOP GOALS AND OUTCOMES}

This workshop will tackle critical issues around social media accessibility, producing artifacts to aid both platform designers and researchers. Workshop participants will listen to the perspectives of social media users with disabilities, and practitioners who can describe their development workflows for accessible products. They will also share their work, form small teams, and be tasked with creating guidelines for researchers and industry practitioners. The guidelines will detail the highest priority accessibility challenges and solutions to ensure these platforms are accessible in future designs. After the workshop, we will publish and promote these guidelines and workshop papers on the workshop website and other venues. These accessibility guidelines will be useful artifacts not only for the CSCW community, but everyone developing or using social media platforms.

\section{WORKSHOP LOGISTICS}

Our proposal is for a single-day workshop on Sunday, November 10th. Other than standard audio/video equipment (projector and screen), we do not anticipate any special equipment or supply needs. We will publicize the workshop and provide access to its content at http://accessiblesocial.media. This includes publishing the call for participation, submission details, as well as the accepted position papers and any guidelines or calls to action produced as a result of the workshop's breakout sessions.

\section{WORKSHOP PARTICIPANTS}

We intend to limit the workshop to a maximum of 30 participants (not counting the organizers). Participants will have two ways to apply for the workshop. First, they may submit a position paper (up to four pages long) relevant to the topic of the workshop. This is especially relevant for researchers who want to detail their current or prior work. Second, they may apply on the workshop website through questionnaires about their experience with accessibility on social media. The application questions will be targeted at people with disabilities and industry professionals.

The workshop organizers will select participants on the basis of their papers or application responses, highlighting provocative position papers or unique accessibility perspectives. We will advertise the workshop and call for position papers via the workshop website, which we will share via email lists for SIGCHI and SIGACCESS, as well as on social media. 


\section{REFERENCES}

[1] Brooke E. Auxier, Cody L. Buntain, Paul Jaeger, Jennifer Golbeck, and Hernisa Kacorri. 2019. \#HandsOffMyADA: A Twitter Response to the ADA Education and Reform Act. In Proceedings of the 2019 CHI Conference on Human Factors in Computing Systems (CHI '19). ACM, New York, NY, USA, Article 527, 12 pages. https://doi.org/10.1145/3290605.3300757

[2] Jeffrey P. Bigham, Ryan S. Kaminsky, Richard E. Ladner, Oscar M. Danielsson, and Gordon L. Hempton. 2006. WeblnSight: Making Web Images Accessible. In Proceedings of the 8th International ACM SIGACCESS Conference on Computers and Accessibility (Assets '06). ACM, New York, NY, USA, 181-188. https://doi.org/10.1145/1168987.1169018

[3] Julian Brinkley and Nasseh Tabrizi. 2017. A Desktop Usability Evaluation of the Facebook Mobile Interface using the JAWS Screen Reader with Blind Users. Proceedings of the Human Factors and Ergonomics Society Annual Meeting 61, 1 (2017), 828-832. https://doi.org/10.1177/1541931213601699 arXiv:https://doi.org/10.1177/1541931213601699

[4] Maria Claudia Buzzi, Marina Buzzi, and Barbara Leporini. 2011. Web 2.0: Twitter and the Blind. In Proceedings of the 9th ACM SIGCHI Italian Chapter International Conference on Computer-Human Interaction: Facing Complexity (CHItaly). ACM, New York, NY, USA, 151-156. https://doi.org/10.1145/2037296.2037333

[5] Amber Ferguson. 2016. The \#CripTheVote Movement Is Bringing Disability Rights To The 2016 Election. (2016). Retrieved August 20, 2018 from https://www.huffingtonpost.com/entry/cripthevote-movement-2016-election_us_ 57279637e4b0f309baf177bd.

[6] Centers for Disease Control and Prevention. 2019. CDC: 1 in 4 US adults live with a disability. (2019). Retrieved June 27, 2019 from https://www.cdc.gov/media/releases/2018/p0816-disability.html.

[7] Cole Gleason, Patrick Carrington, Cameron Cassidy, Meredith Ringel Morris, Kris M. Kitani, and Jeffrey P. Bigham. 2019. "It's Almost Like They're Trying to Hide It": How User-Provided Image Descriptions Have Failed to Make Twitter Accessible. In The World Wide Web Conference (WWW '19). ACM, New York, NY, USA, 549-559. https://doi.org/10.1145/ 3308558.3313605

[8] Darren Guinness, Edward Cutrell, and Meredith Ringel Morris. 2018. Caption Crawler: Enabling Reusable Alternative Text Descriptions Using Reverse Image Search. In Proceedings of the 2018 CHI Conference on Human Factors in Computing Systems (CHI '18). ACM, New York, NY, USA, Article 518, 11 pages. https://doi.org/10.1145/3173574.3174092

[9] Bronwyn Hemsley, Stephen Dann, Stuart Palmer, Meredith Allan, and Susan Balandin. 2015. "We definitely need an audience": experiences of Twitter, Twitter networks and tweet content in adults with severe communication disabilities who use augmentative and alternative communication (AAC). Disability and rehabilitation 37, 17 (2015), 1531-1542.

[10] Amanda Hynan, Juliet Goldbart, and Janice Murray. 2015. A grounded theory of Internet and social media use by young people who use augmentative and alternative communication (AAC). Disability and Rehabilitation 37, 17 (2015), 1559-1575. https://doi.org/10.3109/09638288.2015.1056387 arXiv:https://doi.org/10.3109/09638288.2015.1056387 PMID: 26087813.

[11] Amanda Hynan, Janice Murray, and Juliet Goldbart. 2014. 'Happy and excited': Perceptions of using digital technology and social media by young people who use augmentative and alternative communication. Child Language Teaching and Therapy 30, 2 (2014), 175-186. https://doi.org/10.1177/0265659013519258 arXiv:https://doi.org/10.1177/0265659013519258

[12] Hanlin Li, Disha Bora, Sagar Salvi, and Erin Brady. 2018. Slacktivists or Activists?: Identity Work in the Virtual Disability March. In Proceedings of the 2018 CHI Conference on Human Factors in Computing Systems (CHI '18). ACM, New York, NY, USA, Article 225, 13 pages. https://doi.org/10.1145/3173574.3173799

[13] Hanlin Li and Erin Brady. 2016. \#accessibilityFail: Categorizing Shared Photographs of Physical Accessibility Problems. In Proceedings of the 18th International ACM SIGACCESS Conference on Computers and Accessibility (ASSETS '16). ACM, New York, NY, USA, 277-278. https://doi.org/10.1145/2982142.2982186

[14] Haley MacLeod, Cynthia L. Bennett, Meredith Ringel Morris, and Edward Cutrell. 2017. Understanding Blind People's Experiences with Computer-Generated Captions of Social Media Images. In Proceedings of the 2017 CHI Conference on 
Human Factors in Computing Systems (CHI '17). ACM, New York, NY, USA, 5988-5999. https://doi.org/10.1145/3025453. 3025814

[15] Meredith Ringel Morris, Jazette Johnson, Cynthia L. Bennett, and Edward Cutrell. 2018. Rich Representations of Visual Content for Screen Reader Users. In Proceedings of the 2018 CHI Conference on Human Factors in Computing Systems (CHI '18). ACM, New York, NY, USA, Article 59, 11 pages. https://doi.org/10.1145/3173574.3173633

[16] Meredith Ringel Morris, Annuska Zolyomi, Catherine Yao, Sina Bahram, Jeffrey P. Bigham, and Shaun K. Kane. 2016. "With Most of It Being Pictures Now, I Rarely Use It": Understanding Twitter's Evolving Accessibility to Blind Users. In Proceedings of the 2016 CHI Conference on Human Factors in Computing Systems (CHI '16). ACM, New York, NY, USA, 5506-5516. https://doi.org/10.1145/2858036.2858116

[17] Imke Niediek. 2016. Don't Write It, Picture It!: Accessible Information by Graphic Signs. In Proceedings of the 7th International Conference on Software Development and Technologies for Enhancing Accessibility and Fighting Info-exclusion (DSAI 2016). ACM, New York, NY, USA, 188-193. https://doi.org/10.1145/3019943.3019971

[18] Lindsay Reynolds and Shamoei Wu. 2018. "I'm Never Happy with What I Write": Challenges and strategies of people with dyslexia on social media. In Proceedings of the Twelfth International AAAI Conference on Web and Social Media (ICWSM 18). https://www.aaai.org/ocs/index.php/ICWSM/ICWSM18/paper/view/17812

[19] Shaomei Wu and Lada A. Adamic. 2014. Visually Impaired Users on an Online Social Network. In Proceedings of the SIGCHI Conference on Human Factors in Computing Systems (CHI '14). ACM, New York, NY, USA, 3133-3142. https: //doi.org/10.1145/2556288.2557415

[20] Shaomei Wu, Lindsay Reynolds, Xian Li, and Francisco Guzmán. 2019. Design and Evaluation of a Social Media Writing Support Tool for People with Dyslexia. In Proceedings of the 2019 CHI Conference on Human Factors in Computing Systems (CHI '19). ACM, New York, NY, USA, Article 516, 14 pages. https://doi.org/10.1145/3290605.3300746

[21] Shaomei Wu, Jeffrey Wieland, Omid Farivar, and Julie Schiller. 2017. Automatic Alt-text: Computer-generated Image Descriptions for Blind Users on a Social Network Service. In Proceedings of the 2017 ACM Conference on Computer Supported Cooperative Work and Social Computing (CSCW '17). ACM, New York, NY, USA, 1180-1192. https://doi.org/10. $1145 / 2998181.2998364$ 